\title{
Arbeitstagung der Schweizerischen Gesellschaft für Präventivmedizin und der Schweizerischen Gesellschaft für Soziologie
}

16. Januar $1969 \mathrm{~lm}$ Institut für Sozial- und Präventivmedizin der Universität Zürich

Vorwort

Die Arbeitstagung der Gesellschaften für Soziologie und Präventivmedizin sollte den Vertretern der Soziologie und der Sozial- und Präventivmedizin Gelegenheit geben, ein interdisziplinäres Gespräch über aktuelle medizinische Probleme zu führen. Gegenstand der Diskussionen über die Methodologie bildeten soziologische und sozialmedizinische Studien, die im Hinblick auf diesen Anlaß geplant und durchgeführt, in den meisten Fällen aber noch nicht ausgewertet oder abgeschlossen wurden.
Die folgenden Berichte sind demnach unvollständig und nicht als wissenschaftliche Publikationen, sondern als vorläufige Mitteilungen zu betrachten.

Die Diskussionsbeiträge zu den einzelnen Themen können aus Platzgründen nicht einzeln, sondern nur summarisch wiedergegeben werden. Höhepunkt der Veranstaltung, die von rund 100 Ärten und Soziologen besucht war, bildete das Referat des Gastreferenten, Prof. M. Pflanz, Leiter des Institutes für Epidemiologie und Sozialmedizin der Universität Hannover.

M. Schär 